\title{
High Integrity Localization With Multi-Lane Camera Measurements
}

\author{
Joelle Al Hage, Philippe Xu and Philippe Bonnifait
}

\begin{abstract}
Localization with high integrity is crucial for highly autonomous vehicles. This requires that the localization system send a warning to a client application when it should not be used. The concept of integrity was firstly developed for aviation applications and recently became an active research area for autonomous vehicles. GNSS information merged with dead reckoning sensors is not sufficient for lane level localization in all navigation environments. Map-aided localization with vision sensors is essential to provide redundant and complementary information. In this work, a multi-sensor data fusion method that takes advantage of a high definition (HD) map is presented and the integrity of the obtained solution is quantified. A Fault Detection and Exclusion (FDE) step is added to exclude the faulty measurements from the fusion procedure. A second step is to bound the estimation errors in the Along Track (AT) and Cross Track (CT) directions through Protection Levels (PL). For this step, the usual Gaussian distribution is replaced by a Student's distribution with an adapted degree of freedom chosen according to the navigation environment. The performance of the approach is evaluated with an experimental vehicle equipped with a camera able to detect up to four lane markings simultaneously.
\end{abstract}

\section{INTRODUCTION}

Within the scope of this paper, autonomous driving includes levels 3 to 5 as defined by the Society of Automotive Engineers (SAE) in Standard J3016. That is when the vehicle can navigate autonomously either under certain conditions or without any constraint. Hence, high automation (levels 3 and 4) and full automation (level 5) require the ability of the car to give warning or to make a safe decision in case of a problem which is related to the integrity concept.

Reliable and accurate positions are an essential part for autonomous vehicles in order to navigate safely. Different sensors can be used like wheel speed sensors, gyros, GNSS, LiDARs and cameras. Low-cost GNSS receivers available currently in the market are not adapted for lane level localization especially in urban environments. Therefore, autonomous navigation cannot rely only on these kinds of sensors.

Localizing autonomous vehicles is generally associated with High Definition (HD) road maps that are used to retrieve accurate information for the navigation task [1], [2]. Associating an estimated position obtained from GNSS or dead-reckoning with roads map is done via a map matching procedure [3], [4]. Additional information from exteroceptive sensors (e.g. cameras) can be used to detect lane markings and enhance the positioning output [5], [6].

The authors are with Heudiasyc, UMR 7253, Universite de Technologie de Compiègne, Sorbonne Universités, France. The authors are also members of SIVALab, a joint laboratory between Renault/Heudiasyc/CNRS/UTC.
Integrity is an emergent concern for road localization. Indeed, localization integrity was initially developed for aeronautical applications in order to provide a measure of confidence for GNSS-based navigation solutions [7][9]. It is associated with a target integrity risk which represents the maximum probability that the error in position exceeds a given limit without warning the user. This limit is known as the Protection Level (PL). Aeronautical integrity methods cannot be applied to road vehicles for different reasons [10]. Indeed, they make the assumption of Gaussian distribution with zero mean and they suppose the occurrence of one fault at a time. These hypotheses verified in open sky environments cannot be generalized to urban applications because of multipath and non-line of sight signals which are very frequent in such environments [11], [12]. Moreover, the PL obtained for aeronautical applications are too conservative to be applied to autonomous vehicles. Indeed, most airways are eight nautical miles (14 kilometers) wide while lane width for road vehicles is about $3.5 \mathrm{~m}$.

In this paper, we propose a method for lane level localization for autonomous vehicles with integrity study. For this purpose dead-reckoning measurements are merged with GNSS measurements (GPS and GLONASS) obtained from a low-cost mono-frequency receiver. A smart camera returns measurements of detected lane markings that are used to enhance the localization procedure after associating with a prior HD map. The enhancement is especially done in the cross track direction.

Measurements from sensors are affected by errors where the origin is the sensors or the environmental noises. These errors have a direct effect on the integrity of the localization if they are not detected and excluded from the fusion procedure. Therefore, a Fault Detection and Exclusion (FDE) step is added in order to test the consistency of the GNSS and the camera measurements with the dead-reckoning measurements. A detected error on the camera measurements can get its origin from the camera or from the map. In a second step, the purpose is to bound the errors and to compute a PL after removing all the detected faults. For this step, the Gaussian distribution is replaced by a Student's $t$-distribution which is heavy tailed and thus permits to take into account the errors not considered when using a Gaussian distribution [10]. Likewise, with this distribution, we aim to avoid an undervaluation of the PL. Since we work with autonomous vehicles, it is interesting to express the PL in AT and CT directions; this allows to visualize the benefit of the camera in reducing the PL values in the CT direction.

This paper is organized as follows. Section 2 presents the system modeling and the multi-sensor data fusion. The 
integrity study divided into FDE and error bounding is presented in section 3. Section 4 shows the experimental study and the performance of the method on a recorded dataset in Rambouillet, France. Conclusion and future work are given in section 5 .

\section{SYSTEM MODELING}

The system uses wheel speed sensors, a gyro, GNSS, an intelligent camera for lane markings detection and an HD map. The localization is defined relatively to a Cartesian ENU frame (East, North, Up) denoted $R_{O}$ (figure 1). The origin of this frame can be any position close to the navigation area. Other working frames are needed, and they are shown in figure $1 . R_{B}$ is the body frame located in the middle of the rear wheel axis of the vehicles where the vehicle pose $(x, y, \theta)$ is defined. The detected lanes are expressed in a virtual frame $R_{M}$ located at the center of the front bumper while the camera is located behind the windscreen. The GNSS fix is obtained relatively to the antenna (frame $R_{G}$ ) which is translated with a lever arm relatively to the body frame.

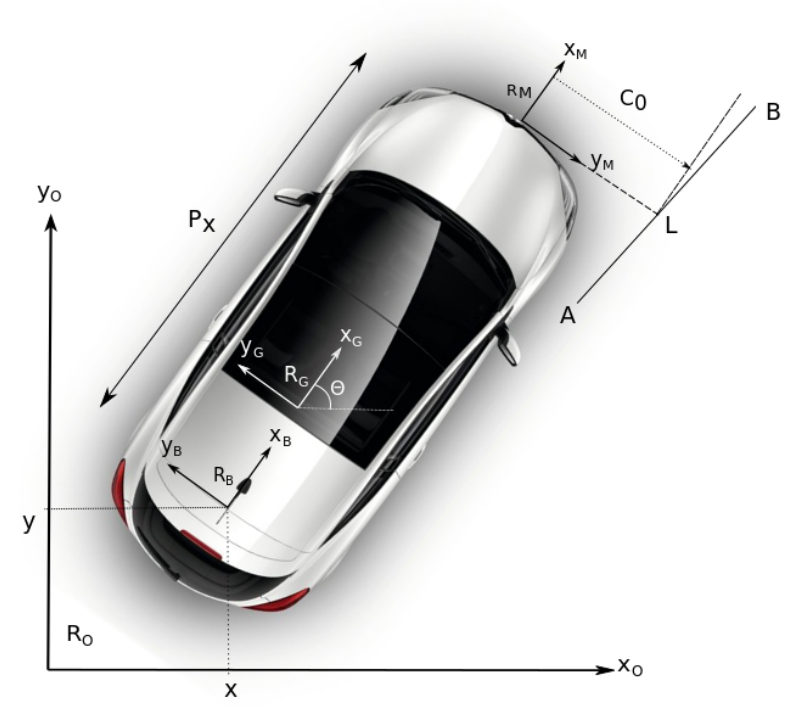

Fig. 1. Frames representation.

The lanes in the map are represented as polylines with an absolute accuracy of the order of a few centimeters. A view of the map is given in figure 2 .

At instant $k$, the state vector is considered to be the position and the heading of the vehicle:

$$
X=\left[\begin{array}{lll}
x & y & \theta
\end{array}\right]^{T}
$$

The GNSS observation is given by:

$$
Z_{1}=\left[\begin{array}{c}
x_{\mathrm{GNSS}} \\
y_{\mathrm{GNSS}}
\end{array}\right]=\left[\begin{array}{c}
t_{x} \cos \theta-t_{y} \sin \theta+x \\
t_{x} \sin \theta+t_{y} \cos \theta+y
\end{array}\right],
$$

where $t_{x}$ and $t_{y}$ are the translation of the antenna with respect to the body frame.

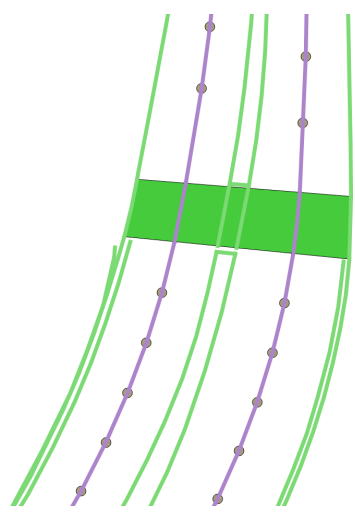

Fig. 2. Detailed view of an HD map. The lane markings are in green, the centerline is in purple, pedestrian crossing is the rectangle in green.

A smart camera detects lane markings and returns the coefficients of a Taylor's expansion of a clothoid in the frame $R_{M}[13]$ :

$$
y=C_{3} x^{3}+C_{2} x^{2}+C_{1} x+C_{0}
$$

The camera is able to detect up to two markings at a time on each side: right and left. In this paper, we consider only the use of the parameter $C_{0}$ (the lateral distance between the lane marking and the frame $R_{M}$ ) to enhance the localization procedure:

$$
Z_{2, j}=C_{0}
$$

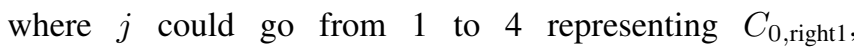
$C_{0, \text { right } 2}, C_{0, \text { left1 }}, C_{0, \text { left2 }}$ if they exist.

The camera observation model was developed in [5], [13] and can be written as:

$C_{0}=\frac{\left(P_{x} \sin \theta+y-y_{A}\right) \cdot x_{A B}-\left(P_{x} \cos \theta+x-x_{A}\right) \cdot y_{A B}}{x_{A B} \cdot \cos \theta+y_{A B} \cdot \sin \theta}$

where the lane marking is detected on the segment $[A B]$ at coordinates $\left[x_{L}, y_{L}\right]$ expressed in frame $R_{O}$ as:

$$
{ }^{O} L={ }^{O} T_{B} \cdot{ }^{B} T_{M} \cdot{ }^{M} L
$$

${ }^{O} T_{B}$ and ${ }^{B} T_{M}$ are the transformation matrices from $R_{B}$ to $R_{O}$ and from $R_{M}$ to $R_{B}$, respectively. Therefore,

$$
\left[\begin{array}{l}
x_{L} \\
y_{L}
\end{array}\right]=\left[\begin{array}{c}
P_{x} \cos \theta+C_{0} \sin \theta+x \\
P_{x} \sin \theta-C_{0} \cos \theta+y
\end{array}\right]
$$

where ${ }^{M} L=\left[0, C_{0}\right]$ and $P_{x}$ is the distance between $R_{B}$ and $R_{M}$ (view figure 1 ).

For state estimation, an extended information filter is used. The information matrix and information vector are updated as follows [14]:

$$
\begin{aligned}
& Y_{k / k}=Y_{k / k-1}+\sum_{i=1}^{n} I_{i, k}, \\
& y_{k / k}=y_{k / k-1}+\sum_{i=1}^{n} i_{i, k},
\end{aligned}
$$

with $n$ the number of observations at instant $k$ and

$$
\begin{aligned}
I_{i, k} & =H_{i, k}^{T} R_{i, k}^{-1} H_{i, k} \\
i_{i, k} & =H_{i, k}^{T} R_{i, k}^{-1}\left[\left(Z_{i, k}-\hat{Z}_{i, k}\right)+H_{i, k} X_{k / k-1}\right] .
\end{aligned}
$$


$\left(I_{i, k}, i_{i, k}\right)$ are the information contributions of the observation $i$, that could be the GNSS measurements or the camera observations (left or right side).

$H_{i}$ is the observation matrix defined as a Jacobian.

$R_{i}$ is the covariance associated to observation noise $Z_{i}$.

It should be noted that the evolution model is obtained from the odometric model using the wheel speed sensors and the gyro [10]. The information matrix and the information vector are obtained as:

$$
\begin{aligned}
Y_{k+1 / k} & =P_{k+1 / k}^{-1} \\
y_{k+1 / k} & =Y_{k+1 / k} X_{k+1 / k}
\end{aligned}
$$

with $P_{k+1 / k}$ the covariance matrix.

The simple summations that appear in equations (7) and (8) are well adapted for multi-sensor data fusion as well as for multi-fault detection and exclusion.

\section{MUlTi-SENSOR DATA FUSION WITH INTEGRITY}

\section{A. Fault detection and exclusion}

The observations from the GNSS receiver or from the camera can be erroneous or faulty. The origin of the fault can come from the sensor, the map or the environmental conditions. Erroneous measurements, if not detected, affect directly the localization procedure. Hence, a FDE step is added where the Mahalanobis distance between the predicted state and the updated state is used as a residual:

$$
r_{k}=\left(X_{k / k}-X_{k / k-1}\right)^{T} Y_{k / k}\left(X_{k / k}-X_{k / k-1}\right)
$$

where $X_{k / k}$ and $Y_{k / k}$ are obtained from a filter that uses all available measurements at instant $k$. Please note that this residual acts in the state space and detects faults which have a direct influence on the position estimate (it is not an innovation gating as classically done). A fault is detected if $r_{k}$ exceeds a threshold value obtained from a Chi-squared distribution with 3-degrees of freedom according to a given false alarm probability.

For the exclusion step, residuals that each use

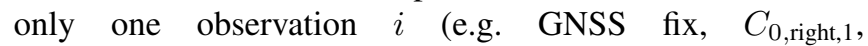
$\left.C_{0, \text { left }, 1}, C_{0, \text { right, } 2}, C_{0, \text { left, } 2}\right)$ are generated:

$$
r_{i, k}=\left(X_{k / k, i}-X_{k / k-1}\right)^{T} Y_{k / k, i}\left(X_{k / k, i}-X_{k / k-1}\right)
$$

where $X_{k / k, i}$ and $Y_{k / k, i}$ are obtained from a filter that uses only the observation $i$ in the update step. Using these residuals, it is possible to distinguish between GNSS and camera measurement errors (left and right).

The architecture of the multi-sensor data fusion with FDE step is given in figure 3 and a summary is given in the following.

At instant $k$, if observations are available (GNSS or camera), the residual for the fault detection can be computed using the informational contributions of the different available measurements (equation 13). If this residual exceeds the threshold value, a fault is detected and its origin has to be determined in order to proceed to its exclusion from the fusion procedure. Therefore, the set of residuals $r_{i, k}(i \in$ $\left.\left\{\mathrm{GNSS}, C_{0, \text { left }, 1}, C_{0, \text { left, } 2}, C_{0, \text { right }, 1}, C_{0, \text { right, } 2}\right\}\right)$ is generated by creating a bank of information filters $\left(E I F_{i}\right)$ where each one uses one observation. Notice that the complete set of residuals is not always available since the sensors measurements are introduced in an asynchronous manner. Moreover, the availability of the camera measurements depends on the navigation environment. The camera gives an indication of the quality of the lane marking detection. Therefore, a lane marking with a quality below a given value is rejected.

A measurement $i$ is excluded if the corresponding residual exceeds the threshold value. For example, if $r_{\text {gnss }}>$ threshold, the GNSS measurements are excluded from the fusion procedure by subtracting their information contributions from the main filter.

Given that the camera is able to detect up to two lane markings on the right or left side, the redundancy can be used to differentiate between camera and map faults which is useful to detect map errors. Let us take the following example to illustrate this. At instant $k$, suppose the camera observes simultaneously $C_{0, \text { left, } 1}$ and $C_{0, \text { left, } 2}$. If $r_{C_{0} \text {,left, } 1}$ obtained from the first observation exceeds the threshold value, we can conclude that a problem appears on this measurement. However, we cannot specify if the error comes from the camera or from the map. Suppose now that the residual $r_{C_{0} \text {,left, } 2}$ is below the threshold. In this case, at the same instant and on the same side, the camera gives one good measurement and one bad measurement. In these conditions, it is likely that the error comes from the map since the second measurement, almost in the same environment, is error free and detected with high confidence. A flag can be stored in the map to indicate that the map-matched segment is potentially erroneous. Now, if $r_{C_{0} \text {, left, } 2}$ is greater than the threshold, it cannot be concluded that there is or not an error in the map. Indeed, if the two observations on the same side are faulty, the error may get its origin from the environment or from the camera itself. Likewise, if only one measurement is available on a given side, the differentiation between the camera and the map is not possible. Notice that the origin of the error (camera or map) does not affect the exclusion procedure. In other words, if $r_{C_{0}, j}$ indicates the presence of a fault, the measurement $C_{0, j}$ will not be used in the update step independently from the origin of the camera measurement error.

Finally, if all the residuals $r_{i}$ indicate the presence of faults, the hypothesis of a filtering problem or deadreckoning problem is likely and an alarm has to be raised.

After determining the origin of the faults (GNSS, $\left.C_{0, \text { left, } 1}, \ldots\right)$, the faulty measurements are excluded from the fusion procedure by subtracting their information contributions from equations 7 and 8 .

\section{B. Protection level computation}

After removing the faulty measurements, the objective is now to bound the errors. The aim is to avoid an underestimation of the PL that may be due to an undervaluation of the covariance matrix of the filter or from undetected biases. Therefore, when computing the 


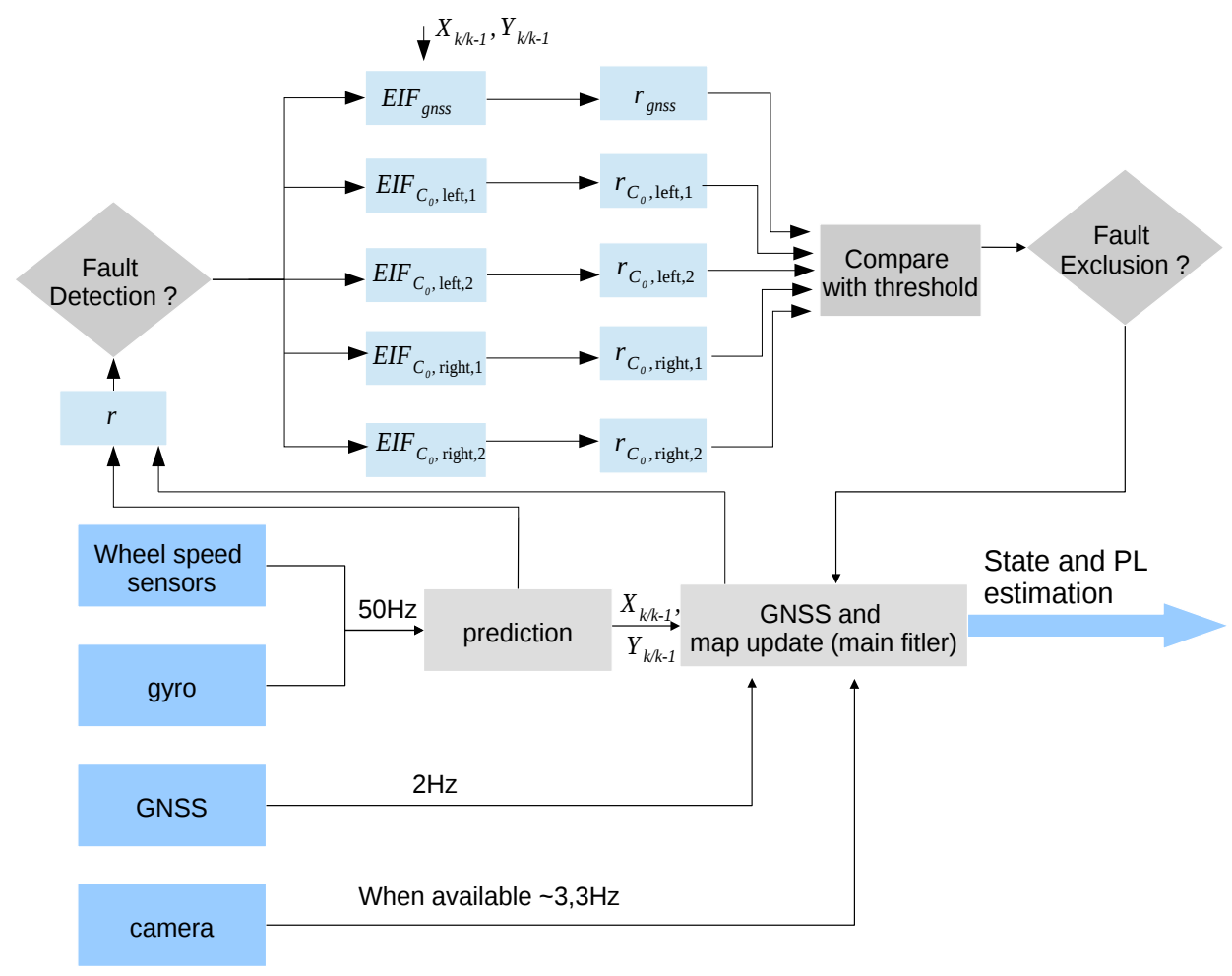

Fig. 3. Architecture of the method to compute an accurate localization with protection levels.

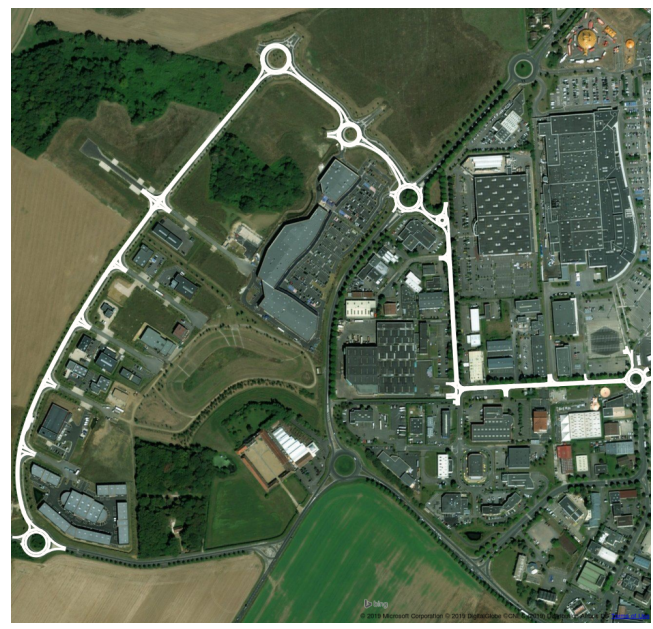

Fig. 4. Experimental environment (Rambouillet, France). The path of the experimental vehicle is displayed in white.

PL, the multivariate Gaussian distribution is replaced by a multivariate Student's distribution which has a heavier tail compared to the latter [10], [15]. For this purpose, the matrix $P_{k / k}$ will be considered as the covariance matrix of a multivariate Student's $t$-distribution with a fixed degree of freedom $N$. Notice that when $N \rightarrow+\infty$, the $t$-distribution converges to a Gaussian one. For more details about the properties of the $t$-distribution, the reader can refer to [10].

The horizontal protection level is expressed as follows
[16]:

$$
P L_{k}(\alpha)=K(\alpha, N) \sqrt{N-2} \sqrt{\max \left(\text { eigenvalue }\left(P_{k / k}\right)\right)},
$$

where $K$ is obtained from a multivariate $t$-distribution with degree of freedom $N$ and according to a given confidence level $\alpha$. The $K(\alpha, N)$ value can be computed by solving numerically the following equation [16]:

$$
\alpha=\frac{2}{B\left(\frac{d}{2}, \frac{N}{2}\right)} \int_{K}^{\infty} \frac{y^{d-1}}{\left(1+y^{2}\right)^{\frac{N+d}{2}}} d y .
$$

where $B\left(\frac{d}{2}, \frac{N}{2}\right)$ is the Beta distribution and $d$ is the dimension of the $t$-distribution; for horizontal $P L, d=2$.

For road vehicles, we are interested by protection levels in the AT and CT directions. Therefore, the covariance matrix in the ENU frame is projected to the (AT, CT) frame using equation 17 :

$$
P_{k / k, p r o j}=R_{\text {proj }} P_{k / k(1,2),(1,2)} R_{\text {proj }}^{T}
$$

with

$$
R_{\text {proj }}=\left[\begin{array}{cc}
\cos \theta & \sin \theta \\
-\sin \theta & \cos \theta
\end{array}\right]
$$

where $\theta$ is the orientation of the vehicle.

The AT and CT PLs are obtained as:

$$
P L_{A T}=K(\alpha, N) \cdot \sqrt{N-2} \sqrt{\left[\text { eigenvalue }\left(P_{k / k, p r o j}\right)\right]_{1}}
$$


TABLE I

$$
P L_{C T}=K(\alpha, N) \cdot \sqrt{N-2} \sqrt{\left[\text { eigenvalue }\left(P_{k / k, p r o j}\right)\right]_{2}}
$$

where the indexes 1 and 2 denote the first and second elements of the vector (eigenvalue $\left(P_{k / k, p r o j}\right)$ ).

\section{EXPERIMENTAL RESULTS}

For the evaluation of the approach, an experiment was carried out in the streets of Rambouillet, France (figure 4), using an experimental vehicle equipped with Ublox $8 \mathrm{~T}$ which is a single frequency multi-constellation GPS/GLONASS receiver, a NovAtel SPAN-CPT with network RTK corrections used as a ground truth and a Mobileye camera for lane marking detections.
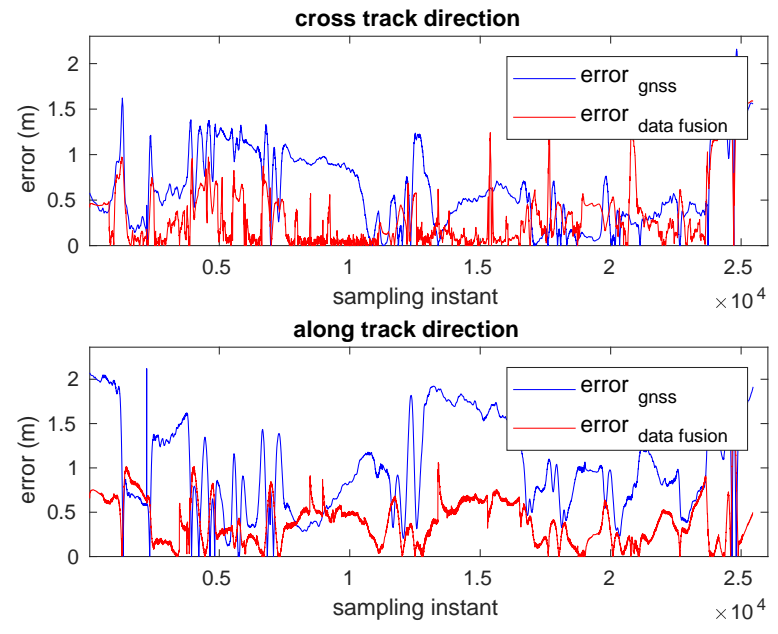

Fig. 5. Errors after data fusion compared to GNSS (sampling period of $0.02 \mathrm{~s})$.
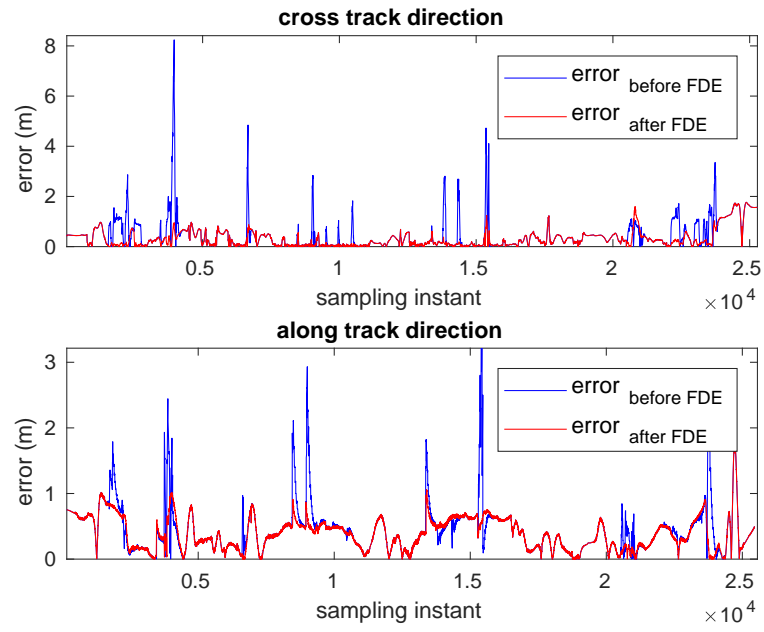

Fig. 6. Errors before and after the FDE step.

The positioning errors in the AT and CT directions after the data fusion and after the FDE step are compared to the
STATISTICAL VALUES OF THE ERRORS BEFORE THE FDE STEP.

\begin{tabular}{|c|c|c|c|}
\hline error (m) & Horizontal & CT & AT \\
\hline Root mean square & 0.72 & \multicolumn{2}{|c|}{} \\
\cline { 1 - 2 } Median & & -0.014 & 0.144 \\
\cline { 1 - 2 } \cline { 4 - 4 } Min (absolute value) & & $2.42 \times 10^{-5}$ & $2.38 \times 10^{-5}$ \\
\cline { 1 - 1 } \cline { 3 - 4 } Max (absolute value) & & 4.34 & 3.54 \\
\hline
\end{tabular}

TABLE II

STATISTICAL VALUES OF THE ERRORS AFTER THE FDE STEP.

\begin{tabular}{|c|c|c|c|}
\hline error (m) & Horizontal & CT & AT \\
\hline Root mean square & 0.585 & & \\
\hline Median & & -0.016 & 0.091 \\
\cline { 1 - 2 } Min (absolute value) & $0.31 \times 10^{-5}$ & $1.81 \times 10^{-5}$ \\
\cline { 1 - 1 } Max (absolute value) & & 1.76 & 1.80 \\
\hline
\end{tabular}

case when using only the GNSS as shown in figure 5. It can be seen that the improvement is more significant in the CT direction where the errors are most of the time very small. This shows the importance of lane marking detections in improving the localization in this direction. The sampling instant corresponds to the frequency of the dead-reckoning $(0.02 \mathrm{~s})$.

Figure 6 shows the errors before and after the FDE step. The effect of the undetected errors on the position estimates is noticeable. Indeed, the surges that appear before the FDE step (in blue) in the CT and AT directions disappear after the FDE step (in red).

Some statistical values of the error before and after the FDE are shown in tables I and II respectively. One can see the reduction in the maximum errors values in the AT and CT directions after the FDE step.

A small part of the trajectory is given in figure 7 . The estimated trajectory is displayed with the ground truth and the map. Note that the map matching algorithm used in this paper is a point to curve matching [3].

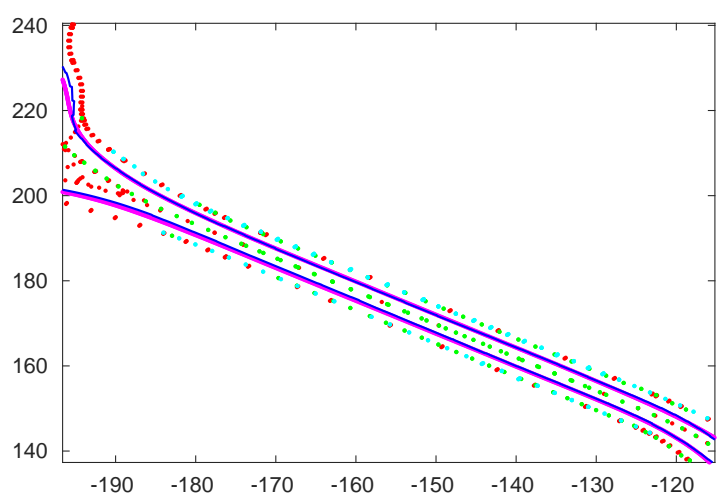

Fig. 7. Zoom in a part of the trajectory: estimated trajectory (blue), ground truth (magenta), map (node and shape point) (red), matched points on the map (green and cyan). Scales in meters.

The residual used for the fault detection is shown in figure 8 . When $r$ indicated the presence of faults, the residuals used for the fault exclusion are generated (if available) and are 


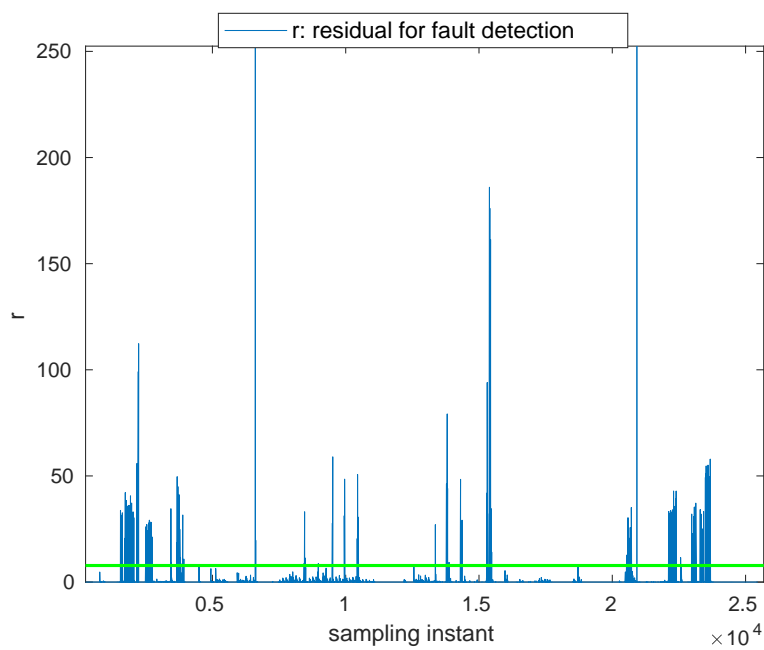

Fig. 8. Residual for the fault detection, in green the threshold value.

displayed in figure 9. Figure 9a shows the residual associated to the GNSS measurements, and figures $9 \mathrm{~b}$ and $9 \mathrm{c}$ show the residuals associated to the camera measurements in the left and right sides, respectively. When two measurements are available simultaneously on the same side, the residual associated to the second measurement is displayed in red.

If a fault is detected on a camera measurements, a visualization of the possible origin of the fault is presented in figure 10. The blue value indicates that the error probably comes from the map, the red value indicates that the origin of the error cannot be specified for one of the two reasons presented before. Figure 11 shows two areas where possible faults have been detected on the map. These detected faults truly correspond to map errors. Indeed, the Mobileye provided two detections on the left side (the link border and the centerline) while only the link border is present on the map which led to an incorrect association.

Let us study the computation of the PL in the AT and CT directions. As we have mentioned before, the Student's $t$-distribution replaces the Gaussian distribution for this step. To adjust the degree of freedom of the $t$-distribution, data acquisitions with a ground truth system are needed in the target environment (urban, suburban, open sky, etc.) [10]. The experiment described in this paper has been done in a suburban area. Accordingly, the degree of freedom of the $t$-distribution is fixed to $N=6$ to preserve a heavy tail necessary to obtain a consistent PL. The target integrity risk is fixed to $10^{-3}$ since we cannot test with a smaller value given the available number of samples. The performance of the computed PL in the AT and CT directions are shown in figure 12. It can be seen that the perception sensor used for lane markings detection reduces the PL in the CT direction down to $1.05 \mathrm{~m}$. The intervals where the PLs in the CT direction show an increase correspond to situations where the vehicle is on a roundabout area. Indeed, the camera cannot detect markings on these kinds of trajectories because of its limited field of view. Therefore, in these areas, the localization is done using only dead-reckoning and GNSS which results in a direct augmentation in the computed PL. The PL in the AT direction is about $2.5 \mathrm{~m}$. To further reduce this value, the camera should be used to detect also information like pedestrian crossing or traffic signs which has not been done in this work.

It should be noted that the computation of the PLs was consistent since the measured frequency of errors that exceeded the PL was lower than the fixed integrity risk: $P($ error $>P L)=0<10^{-3}$ in the AT, CT and horizontal directions. This is not the case if the Gaussian distribution is used where the measured frequencies of errors that exceed the PL are $4.32 \times 10^{-4}, 0.0035$ and 0.0012 in AT, CT and horizontal directions, respectively.

\section{CONCLUSION AND FUTURE WORK}

In this work, we have proposed a localization method that exploits lane features for autonomous vehicles with integrity considerations. For this purpose, a FDE strategy has been proposed. It takes advantage of different types of sensors measurements present in the system: GNSS, deadreckoning, Mobileye for lane markings detection and an HD map. A bank of information filters was used to determine the origin of the faults after creating a set of residual tests. The obtained results confirm the importance of the FDE step as it improves significantly the localization accuracy. In a second step, we have proposed a method to bound in a consistent manner the errors by replacing the Gaussian distribution with a Student's distribution. The PLs are computed in the AT and $\mathrm{CT}$ directions in order to give a concrete meaning of PL for classical road vehicles navigation tasks like path following. The obtained PL is about $1.05 \mathrm{~m}$ in the CT direction (when data from the perception sensor are available) and about 2.5 $m$ in the AT direction.

In future work, the use of other perception sensors like velodyne LiDAR will be investigated in order to improve the results, especially on roundabouts.

\section{ACKNOWLEDGMENTS}

This work was carried out in the framework of the Equipex ROBOTEX (ANR-10- EQPX-44-01) and SIVALab, a shared laboratory between Renault and Heudiasyc UMR UTC/CNRS. The authors thank Corentin Sanchez, Anthony Welte, Emmanuel Stawarsky, Stéphane Bonnet and Clément Zinoune for their support in the experiments.

\section{REFERENCES}

[1] D. Betaille and R. Toledo-Moreo, "Creating enhanced maps for lane-level vehicle navigation," IEEE Transactions on Intelligent Transportation Systems, vol. 11, no. 4, pp. 786-798, 2010.

[2] M. A. Quddus, W. Y. Ochieng, and R. B. Noland, "Current mapmatching algorithms for transport applications: State-of-the art and future research directions," Transportation research part c: Emerging technologies, vol. 15, no. 5, pp. 312-328, 2007.

[3] C. E. White, D. Bernstein, and A. L. Kornhauser, "Some map matching algorithms for personal navigation assistants," Transportation research part c: emerging technologies, vol. 8, no. 16, pp. 91-108, 2000.

[4] A. Kasmi, D. Denis, R. Aufrere, and R. Chapuis, "Map matching and lanes number estimation with openstreetmap," in $201821 s t$ International Conference on Intelligent Transportation Systems (ITSC), IEEE, 2018, pp. 2659-2664. 


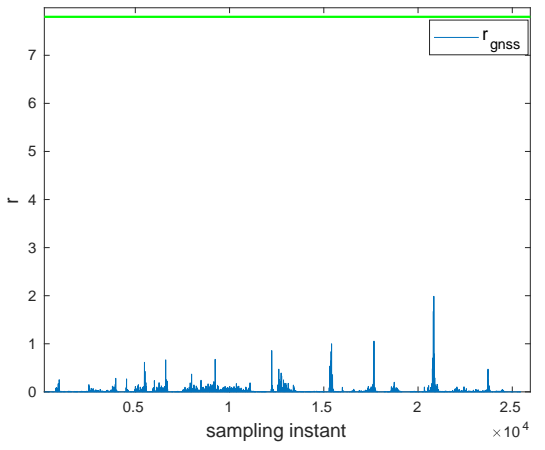

(a) $r_{G N S S}$

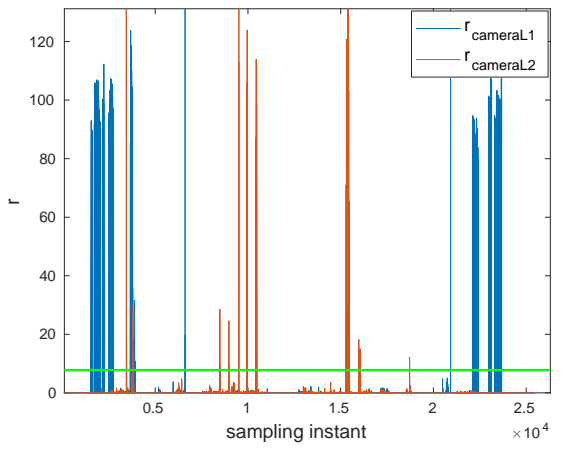

(b) $r_{\text {camera Left }, 1}$ (blue), $r_{\text {camera Left, }, 2}$ (red)

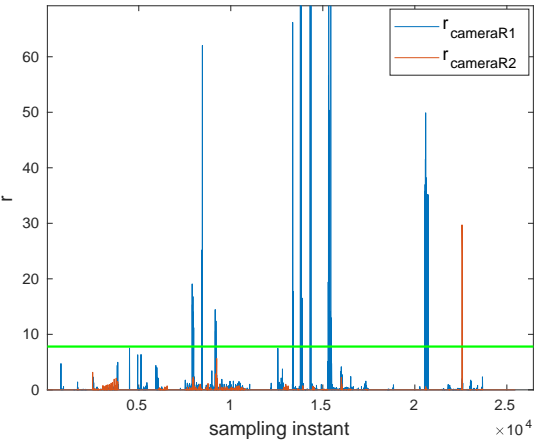

(c) $r_{\text {camera Right ,1 }}$ (blue), $r_{\text {camera Right ,2 }}$ (red)

Fig. 9. Residuals for fault exclusion, in green the thresholds.

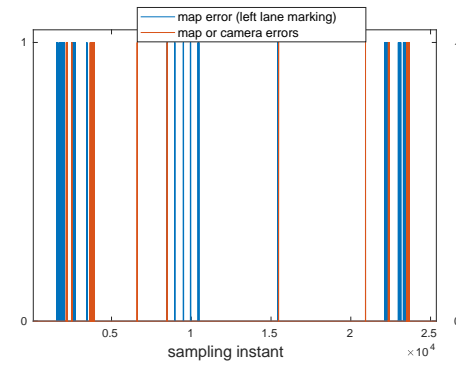

(a) Case 1: left case

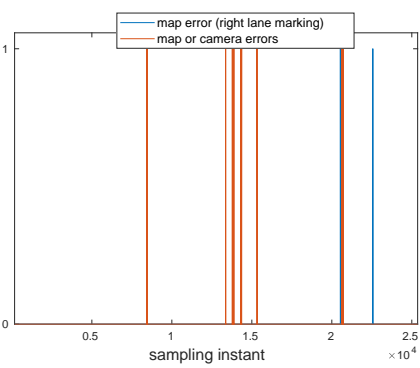

(b) Case 2: right case
Fig. 10. Map error (left or right marking) (blue), camera or map error (cannot specify) (red).

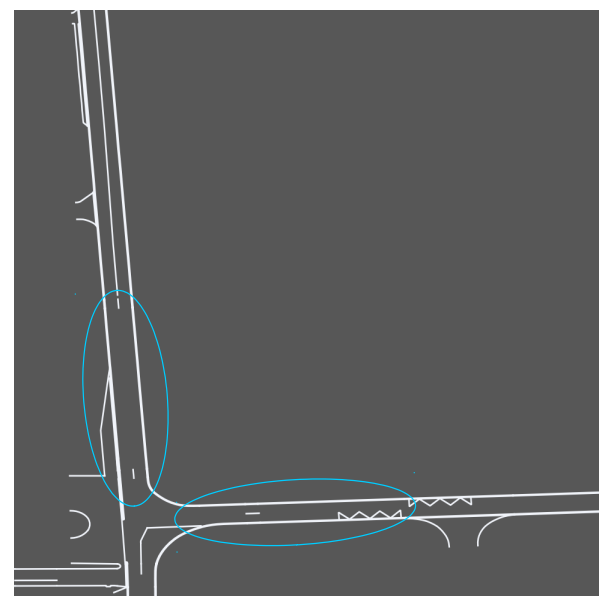

Fig. 11. Areas where map faults have been detected.

[5] Z. Tao, P. Bonnifait, V. Fremont, and J. Ibanez-Guzman, "Mapping and localization using GPS, lane markings and proprioceptive sensors," in IEEE/RSJ International Conference on Intelligent Robots and Systems (IROS 2013), 2013, pp. 406-412.

[6] F. Li, P. Bonnifait, and J. Ibanez-Guzman, "Map-aided deadreckoning with lane-level maps and integrity monitoring," IEEE Transactions on Intelligent Vehicles, vol. 3, no. 1, pp. 81-91, Mar. 2018, ISSN: 2379-8904.

[7] B. W. Parkinson and P. Axelrad, "Autonomous GPS integrity monitoring using the pseudorange residual," Navigation, vol. 35, no. 2, pp. 255-274, 1988.

[8] R. G. Brown, "A baseline GPS RAIM scheme and a note on the equivalence of three RAIM methods," Navigation, vol. 39, no. 3, pp. 301-316, 1992
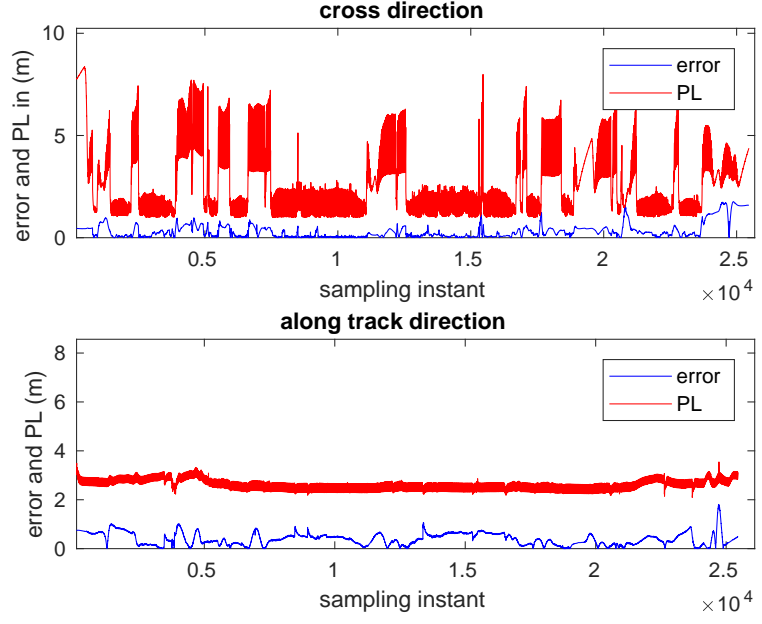

Fig. 12. Errors and PLs in the AT and CT directions.

[9] C. D. S. Andres, "Integrity monitoring applied to the reception of GNSS signals in urban environments," PhD thesis, 2012.

10] J. Al Hage, P. Xu, and P. Bonnifait, "Bounding localization errors with student distribution for road vehicles," in ITSNT 2018 , International Technical Symposium on Navigation and Timing, 2018.

[11] P. D. Groves, Z. Jiang, L. Wang, and M. K. Ziebart, "Intelligent urban positioning using multi-constellation GNSS with $3 \mathrm{~d}$ mapping and NLOS signal detection," 2012.

[12] K. Ali, M. Pini, and F. Dovis, "Measured performance of the application of EGNOS in the road traffic sector," GPS solutions, vol. 16, no. 2, pp. 135-145, 2012.

[13] Z. Tao, P. Bonnifait, V. Fremont, J. Ibanez-Guzman, and S. Bonnet, "Road-centered map-aided localization for driverless cars using single-frequency GNSS receivers," Journal of Field Robotics, vol. 34, no. 5, pp. 1010-1033, 2017.

[14] H. Durrant-Whyte and T. C. Henderson, "Multisensor data fusion," in Springer handbook of robotics, Springer, 2008, pp. 585-610.

[15] A. Dhital, J. B. Bancroft, and G. Lachapelle, "A new approach for improving reliability of personal navigation devices under harsh GNSS signal conditions," Sensors, vol. 13, no. 11, pp. $15221-$ $15241,2013$.

[16] P. F. Navarro Madrid, "Device and method for computing an error bound of a kalman filter based gnss position solution," pat., Apr. 2016. 